\title{
The Robotic Super-LOTIS Telescope: Results \& Future Plans
}

\author{
G. G. Williams*, P. A. Milne ${ }^{\dagger}$, H. S. Park ${ }^{* *}$, S. D. Barthelmy ${ }^{\ddagger}$, \\ D. H. Hartmann ${ }^{\S}$, A. Updike ${ }^{\S}$ and K. Hurley ${ }^{\text {II }}$ \\ ${ }^{*}$ MMT Observatory, P.O. Box 210065, University of Arizona, Tucson, AZ 85721-0065 \\ ${ }^{\dagger}$ Steward Observatory, $933 \mathrm{~N}$ Cherry Ave, Tucson, AZ, 85721 \\ ** Lawrence Livermore National Laboratory, 7000 East Avenue, Livermore, CA 94550 \\ ${ }^{\ddagger}$ NASA Goddard Space Flight Center, Greenbelt, MD 20771 \\ ${ }^{\S}$ Department of Physics and Astronomy, Clemson University, Clemson, SC 29634 \\ "Space Sciences Laboratory, University of California, Berkeley, CA 94720
}

\begin{abstract}
We provide an overview of the robotic Super-LOTIS (Livermore Optical Transient Imaging System) telescope and present results from gamma-ray burst (GRB) afterglow observations using Super-LOTIS and other Steward Observatory telescopes. The 0.6-m Super-LOTIS telescope is a fully robotic system dedicated to the measurement of prompt and early time optical emission from GRBs. The system began routine operations from its Steward Observatory site atop Kitt Peak in April 2000 and currently operates every clear night. The telescope is instrumented with an optical CCD camera and a four position filter wheel. It is capable of observing Swift Burst Alert Telescope (BAT) error boxes as early or earlier than the Swift UV/Optical Telescope (UVOT). Super-LOTIS complements the UVOT observations by providing early R- and I-band imaging. We also use the suite of Steward Observatory telescopes including the 1.6-m Kuiper, the 2.3-m Bok, the $6.5-\mathrm{m}$ MMT, and the 8.4-m Large Binocular Telescope to perform follow-up optical and near infrared observations of GRB afterglows. These follow-up observations have traditionally required human intervention but we are currently working to automate the $1.6-\mathrm{m}$ Kuiper telescope to minimize its response time.
\end{abstract}

Keywords: Super-LOTIS, gamma-ray bursts, afterglows

PACS: $95.55 . \mathrm{Cs}, 98.70 . \mathrm{Rz}$

\section{INTRODUCTION}

The Super-LOTIS collaboration aims to obtain two types of data sets that can be used independently or together to provide a more complete understanding of the physics of GRBs. These data sets are: (1) very early optical imaging, and (2) follow-up optical and near infrared imaging, spectroscopy, and polarimetry. The early optical imaging is provided by a fully robotic 0.6-m telescope and the follow-up data comes from larger aperture Steward Observatory telescopes.

Early-time GRB optical counterpart identification is important because it provides information such as: (1) the peak optical flux, useful for planning follow-up observations; (2) the early decay indices, useful in identifying subsequent flaring activity or breaks in the light curve; (3) color, useful in estimating redshift and planning follow-up observations; and (4) variability, useful in understanding continued activity in the central engine or structure in the circumburster medium [e. g. 1]. Perhaps the most scientifically valuable of these is the variability which manifests itself in different forms that are still not 
TABLE 1. Super-LOTIS characteristics.

\begin{tabular}{lr}
\hline Site: & Steward Observatory, Kitt Peak \\
Aperture: & $0.6-\mathrm{m}$ \\
Instrument: & Spectral Instruments 800 Series Camera \\
Detector: & E2V TE Cooled CCD \\
Format: & $2048 \times 2048,13.5 \mu \mathrm{m}$ pixels \\
Pixel Scale: & $0.5^{\prime \prime} / \mathrm{pix}$ \\
Field-of-View: & $17^{\prime} \times 17^{\prime}$ \\
Filters: & $\mathrm{V}, \mathrm{R}, \mathrm{I}, \mathrm{H}-\alpha$ \\
Limiting Magnitude: & $\mathrm{R} \sim 17.5(10 \mathrm{~s}), \mathrm{R} \sim 18.5(60 \mathrm{~s})$ \\
Slew Speed: & $8^{\circ} / \mathrm{s}$ \\
Response Time: & $<25 \mathrm{~s}$ \\
Software: & Perl Client/Server \\
\hline
\end{tabular}

fully explained.

Follow-up imaging contributes to the long term light curves and yields information about chromatic (spectral evolution) or achromatic (jet) breaks. In addition to providing a redshift, spectroscopy can yield information about the circumburster medium, the intergalactic medium, and in some cases the the emerging supernova. Finally, polarimetry is a powerful tool for probing the nature of the jet and its evolution.

\section{SYSTEM OVERVIEW}

Super-LOTIS is a fully robotic 0.6-m telescope dedicated to the search for optical counterparts of GRBs. The telescope is housed in a roll-off-roof facility at Steward Observatory's Kitt Peak site. From 1999 to 2003 Super-LOTIS was configured with a prime focus imager in order to provide better coverage of the large Burst and Transient Source Experiment (BATSE) error boxes. Selected results from that system and its predecessor, LOTIS, are included in Park et al. [2], Williams et al. [3], Schaefer et al. [4], Park et al. [5], Hurley et al. [6], Castro Cerón et al. [7], and Blake et al. [8].

The upgraded f/9 Cassegrain system uses a commercial Spectral Instruments 800 Series CCD camera equipped with a thinned $2048 \times 2048$ pixel E2V detector. The pixel scale of $0 . / 5 /$ pixel is well matched to the image quality of the optical design and the typical site seeing. The $17^{\prime} \times 17^{\prime}$ field-of-view provides full coverage of the Swift BAT error boxes. The system is linked to the GCN network and can slew at a rapid rate of approximately $8 \%$ which allows the telescope to begin imaging any part of the sky within $25 \mathrm{~s}$ of receiving a GCN trigger. Depending on the observing conditions, the system can often achieve a limiting magnitude of approximately $\mathrm{R}=17.5$ in a single $10 \mathrm{~s}$ exposure and $R=18.5$ in a single 60 s exposure. When not observing GRBs, the system performs ancillary observing programs including nightly multi-band observations of supernovae and searches for novae in M31 and M33.

The Super-LOTIS website, http://slotis.kpno.noao.edu/LOTIS/index.php, provides additional details, status information, and up-to-date results. The characteristics of the system are provided in Table 1 . 


\section{RECENT RESULTS}

Table 2 provides a list of Swift era observations obtained by our group which resulted in GCN Circulars 1 . The table is not a complete log since some of our observations did not result in GCN Circulars. The upper section of Table 2 lists events that were observed by Super-LOTIS and the lower section includes observations using other Steward Observatory facilities.

Super-LOTIS has provided the earliest filtered observations of all eleven bursts that the system responded to promptly. Several of those observations resulted in detections but even the early-deep upper limits provide scientifically interesting constraints. For events that were not observed promptly, either because of timing, position, weather or a delayed trigger, deeper detections or limits were achieved through co-addition of many individual frames.

Since the Swift UVOT is equipped with only blue filters the Super-LOTIS R- \& Iband data complement the Swift UVOT data. The color information can provide crucial clues about the burst environment and early indications of the redshift. In addition, the Super-LOTIS observations occasionally cover gaps in the UVOT coverage which result from pointing or slewing restrictions or periods of Earth blockage. Optical observations are critical during those gaps since flares or breaks in the light curve could occur during them.

\section{FUTURE PLANS}

We plan to continue to operate Super-LOTIS as long as funding permits while at the same time expanding our use of other Steward Observatory telescopes for GRB afterglow observations. Toward this end, we have started the process of automating the 1.6-m Kuiper telescope. The Super-LOTIS operations software was written to be easily ported to the other Steward telescopes. We successfully demonstrated queue mode operation of the Kuiper telescope in October 2007. We are currently assessing the hardware modifications required to remotely control focus, mirror covers, and the dome slit. We anticipate that robotic or human assisted automated operation will be available before the end of 2008.

The twin 8.4-m Large Binocular Telescope (LBT) will begin regular science observing in February 2008. The red and blue channel prime focus cameras for the LBT can detect very faint sources and therefore we plan to use them to obtain very late images to search for supernova bumps, breaks in the light curve, and host galaxies.

\section{ACKNOWLEDGMENTS}

This work was supported in part by NASA Proposal Number 06-SWIFT306-0067.

\footnotetext{
${ }^{1}$ The LBT data were obtained by P. Garnavich and X. Dai as part of the LBT Science Demonstration Time, see Dai et al. [9]
} 
TABLE 2. Recent observations resulting in GCN circulars.

\begin{tabular}{|l|c|c|c|c|}
\hline UT Date & Response Time & Exp. (s) & R Magnitude & GCN Circ. \\
\hline 071025 & $314.8 \mathrm{~s}(95.3 \mathrm{~s})$ & $10 \times 60(10)$ & $17.97 \pm 0.17(>17.3)$ & 6995 \\
071011 & $40.4 \mathrm{~s}$ & 10 & $>16.9$ & 6887 \\
$071010 \mathrm{~b}$ & $13.6 \mathrm{~h}$ & $59 \times 60$ & $>20.4$ & 6893 \\
$070612 \mathrm{a}$ & $25.1 \mathrm{~h}$ & $6 \times 60$ & $17.73 \pm 0.4$ & 6535 \\
070610 & $2.5 \mathrm{~d}$ & $60 \times 60$ & $>20.9$ & 6536 \\
$070419 \mathrm{a}$ & $456 \mathrm{~s}(102 \mathrm{~s})$ & $60(10)$ & $18.49 \pm 0.2(>17.7)$ & 6328 \\
061126 & $35 \mathrm{~s}$ & 10 & $12.9 \pm 0.2$ & 5869 \\
$061102 \mathrm{~b}$ & $38 \mathrm{~s}$ & $5 \times 10$ & $>19.8$ & 5780 \\
061009 & $3.1 \mathrm{~h}$ & $14 \times 60$ & $>20.2$ & 5742 \\
060923 & $41.1 \mathrm{~s}$ & 10 & $>16.6$ & 5588 \\
060515 & $1745 \mathrm{~s}$ & $8 \times 60$ & $>18.1$ & 5136 \\
$060510 \mathrm{~b}$ & $1877 \mathrm{~s}$ & 60 & $>18.6$ & 5100 \\
060502 & $1284 \mathrm{~s}(33 \mathrm{~s})$ & 60 & $>17.9$ & 5049 \\
060501 & $1.25 \mathrm{~h}$ & 60 & $>18.6$ & 5045 \\
060210 & $94.5 \mathrm{~s}(55.1 \mathrm{~s})$ & $5 \times 10(10)$ & $18.25 \pm 0.29(>17.5)$ & 4730 \\
060206 & $4.2 \mathrm{~h}$ & $20 \times 60$ & $17.87 \pm 0.12$ & 4699 \\
060110 & $38.0 \mathrm{~s}$ & 10 & $>16.0$ & 4469 \\
051111 & $35.9 \mathrm{~s}$ & 10 & $13.2 \pm 0.1$ & 4252 \\
$051109 \mathrm{~b}$ & $56.0 \mathrm{~s}$ & 10 & $>17.0$ & 4225 \\
$051109 \mathrm{a}$ & $43.0 \mathrm{~s}$ & 10 & $15.27 \pm 0.13$ & 4218 \\
050525 & $6.0 \mathrm{~h}$ & $20 \times 30$ & $>17.5$ & 3485 \\
050421 & $3.8 \mathrm{~h}(8.0 \mathrm{~h})$ & $30 \times 30$ & $>17.5(20.0)$ & 3311 \\
\hline \hline
\end{tabular}

${ }^{1}$ 1.6-m Kuiper $\quad{ }^{2} 8.4-\mathrm{m} \mathrm{LBT} \quad{ }^{3} 2.3-\mathrm{m}$ Bok

\section{REFERENCES}

1. N. R. Butler, et al., ApJ 652, 1390-1399 (2006), arXiv:astro-ph/0606763.

2. H. S. Park, et al., ApJ 490, L21+ (1997).

3. G. G. Williams, et al., ApJ 519, L25-L29 (1999), arXiv:astro-ph/9902190

4. B. E. Schaefer, et al., ApJ 524, L103-L106 (1999), arXiv : astro-ph/9907235

5. H. S. Park, et al., ApJ 571, L131-L135 (2002), arXiv: astro-ph/ 0112397.

6. K. Hurley, et al., ApJ 567, 447-453 (2002), arXiv: astro-ph/0107188

7. J. M. Castro Cerón, et al., A\&A 393, 445-451 (2002), arXiv : astro-ph/0110049

8. C. Blake, et al., A\&A 399, 365-372 (2003), arXiv: astro-ph/ 0211158.

9. X. Dai, et al., ArXiv e-prints 712 (2007),0712.2239. 